\title{
Gemeinsam forschen für die Zukunft
}

\section{Gastbeiträge der Gewinnerteams des „Best Scientific Analysis Award”}

Das YES! - Young Economic Summit ist einer der größten Schulwettbewerbe in Deutschland rund um wirtschaftliche und gesellschaftliche Herausforderungen. Unter dem Motto „Your future - Your ideas" arbeiten Teams ab der Klassenstufe 10 sowohl eigenständig als auch in Zusammenarbeit mit Forschenden aus Instituten der Wirtschafts- und Sozialforschung an Lösungen zu ökonomischen, ökologischen und gesellschaftlichen Fragen. Die Schüler:innen diskutieren die lokalen und globalen Herausforderungen der Gegenwart und Zukunft. Die Forschungsthemen werden von engagierten Wissenschaftler:innen aus derzeit 30 Forschungsinstituten angeregt. Der „Best Scientific Analysis Award“ ist dabei ein Sonderpreis, der jenen Schulteams verliehen wird, die ihre Lösungsidee besonders wissenschaftlich erarbeitet haben. 2021 sind dies gleich zwei Schulen: das Landschulheim Grovesmühle und die Deutsche Berufsschule Hong Kong. Ihre Ergebnisse werden im Wirtschaftsdienst veröffentlicht.

Das YES! ist ein gemeinsames Projekt der ZBW - Leibniz-Informationszentrum Wirtschaft und der Joachim Herz Stiftung und steht seit 2015 unter der Schirmherrschaft des Bundesministeriums für Wirtschaft und Energie.

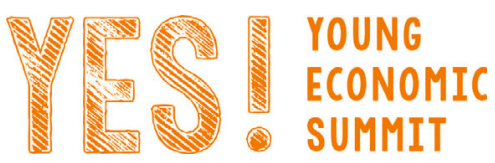

Applaus allein reicht nicht - wie können Pflegeberufe attraktiver werden?

Michaela Fuchs, Institut für Arbeitsmarkt- und Berufsforschung (IAB).

Julia Lang, Institut für Arbeitsmarkt- und Berufsforschung (IAB).

Dominik Mann, Sean-Pascal Kuttner, Julius Georgi Wesirow, Lukas Bachmann, Anna Marie Körner, Yannis Grabbe, Laura Rothe, Lisa Schueler, Landschulheim Grovesmühle.

\section{Steuerehrlichkeit und Steuerlotterien}

Philipp Krug, Katholische Universität Eichstätt-Ingolstadt und KU Research Institute for Taxation.

Dominika Langenmayr, Katholische Universität Eichstätt-Ingolstadt und KU Research Institute for Taxation; WU Wien; CESifo München.

Niklas Rauhut, Finn Ole Schmude, Thea Seitz, Douglas Strasoldo, Moritz Withoeft, Deutsche Berufsschule Hong Kong. 